\title{
Unsteadiness in Flow over a Flat Plate at Angle-of-Attack at Low Reynolds Numbers*
}

\author{
Kunihiko Taira† William B. Dicksonł Tim Colonius§, Michael H. Dickinson` \\ California Institute of Technology, Pasadena, California, 91125 \\ Clarence W. Rowley"l \\ Princeton University, Princeton, New Jersey, 08544
}

\begin{abstract}
Flow over an impulsively started low-aspect-ratio flat plate at angle-of-attack is investigated for a Reynolds number of 300. Numerical simulations, validated by a companion experiment, are performed to study the influence of aspect ratio, angle of attack, and planform geometry on the interaction of the leading-edge and tip vortices and resulting lift and drag coefficients. Aspect ratio is found to significantly influence the wake pattern and the force experienced by the plate. For large aspect ratio plates, leading-edge vortices evolved into hairpin vortices that eventually detached from the plate, interacting with the tip vortices in a complex manner. Separation of the leading-edge vortex is delayed to some extent by having convective transport of the spanwise vorticity as observed in flow over elliptic, semicircular, and delta-shaped planforms. The time at which lift achieves its maximum is observed to be fairly constant over different aspect ratios, angles of attack, and planform geometries during the initial transient. Preliminary results are also presented for flow over plates with steady actuation near the leading edge.
\end{abstract}

\section{Introduction}

In recent years, flows around flapping insect wings have been investigated and, in particular, it has been observed that the leading-edge vortex (LEV) formed during stroke reversal remains stably attached throughout the wing stroke, greatly enhancing lift. ${ }^{1,2}$ Such LEVs are found to be stable over a wide range of angles of attack and for Reynolds number over a range of $R e \approx \mathcal{O}\left(10^{2}\right)-\mathcal{O}\left(10^{4}\right)$. Motivated by the aerodynamic benefits of a stable LEV, a multidisciplinary university research initiative (MURI) has been undertaken to investigate using closed-loop control of actuators along the leading edge and tip of conventional translating airfoils to stabilize the LEV. ${ }^{3,4}$ In the present paper, we investigate the flow structure and resulting forces on low-aspect-ratio flat plate at low Reynolds number. Since most of the research effort in biological flight is directed toward understanding revolving and flapping flights, there is a lack of documentation for translating wings at low Re. There also is a scarcity of three-dimensional studies of flow around low-aspect ratio wings at low $R e$ in general. The lower end of translating flight studies for low aspect ratio wings have been experimentally reported by Torres and Mueller ${ }^{5}$ with $R e \geq 7 \times 10^{4}$. Our

\footnotetext{
*Work supported by a Multidisciplinary Research Initiative from the United States Air Force Office of Scientific Research (FA9550-05-1-0369, Program Manager: Dr. Fariba Fahroo).

${ }^{\dagger}$ Ph.D. Candidate, Mechanical Engineering, Student Member AIAA

${ }^{\ddagger}$ Postdoctoral Scholar, Bioengineering, Member AIAA

$\S$ Professor, Mechanical Engineering, Member AIAA

๑Professor, Bioengineering. Member AIAA

${ }$ Assistant Professor, Mechanical and Aerospace Engineering, Member AIAA
} 
intent is to investigate the general behavior of flow around translating wings at $R e \approx \mathcal{O}\left(10^{2}\right)$ with a number of aspect ratios, angles of attack, and planform geometries.

Both two- and three-dimensional numerical simulations with an immersed boundary method are used to analyze the flow field over flat-plate airfoils. The solution is first validated for flow over a rectangular flat plate of $R=2$ at $R e=100$ with measurements from a companion tow-tank experiment equipped with stereo digital particle image velocimetry (DPIV) capability and a six-axis force sensor. In this study, we define the Reynolds number, $R e$, and aspect ratio of the flat plate, $R$, as $R e \equiv \frac{U_{\infty} \bar{c}}{\nu}$ and $R \equiv \frac{A}{\bar{c}}$, where $U_{\infty}, A$, and $\bar{c}$ are the freestream velocity, the planform area of the plate, and the mean chord of the plate, respectively. For the spacial case of two-dimensional simulations, we express the aspect ratio as $R=\infty$. Once the numerical results are validated, a number of simulations are carried out for a slightly higher Reynolds number of $R e=300$. This Reynolds number is selected as it is past the critical Reynolds number for a sphere, beyond which the flow is unsteady and vortex shedding occurs. Aspect ratio $(R)$, angles of attack $(\alpha)$, and the planform geometry are varied to study their effect on the vortical structure behind the plate and the corresponding lift and drag coefficients. Finally, some preliminary results with modeled leading-edge actuation (steady blowing) are presented. A companion paper ${ }^{4}$ considers reduced-order model with balanced proper orthogonal decomposition method to develop feedback control to stabilize LEV over a plate.

\section{Simulations}

\section{A. Numerical Method}

Simulations of incompressible viscous flow over flat plates are performed with an immersed boundary method. ${ }^{6,7}$ Immersed boundary methods allow the computational domain to be discretized with a nonbody conforming Cartesian grid by applying appropriate boundary force along the immersed surface to satisfy the no-slip condition. We apply a new formulation of the immersed boundary method with a structure algebraically identical to the traditional fractional step method. ${ }^{8}$ This method is based on a finite volume staggered grid discretization and is capable of computing flow over stationary or moving bodies.

The continuous analog of the immersed boundary formulation can be represented by

$$
\begin{aligned}
\frac{\partial \mathbf{u}}{\partial t}+\mathbf{u} \cdot \nabla \mathbf{u} & =-\nabla p+\frac{1}{R e} \nabla^{2} \mathbf{u}+\int_{s} \mathbf{f}(\boldsymbol{\xi}(s, t)) \delta(\boldsymbol{\xi}-\mathbf{x}) d s \\
\nabla \cdot \mathbf{u} & =0 \\
\mathbf{u}(\boldsymbol{\xi}(s, t)) & =\int_{\mathbf{x}} \mathbf{u}(\mathbf{x}) \delta(\mathbf{x}-\boldsymbol{\xi}) d \mathbf{x}=\mathbf{u}_{B}(\boldsymbol{\xi}(s, t))
\end{aligned}
$$

where $\mathbf{u}, p$, and $\mathbf{f}$ are the appropriately non-dimensionalized velocity, pressure, and surface force. The spatial variable in the computational domain $\mathcal{D}$ and along the immersed boundary $\partial \mathcal{B}$ are denoted by $\mathbf{x}$ and $\boldsymbol{\xi}$, respectively. Note that we have written the no-slip boundary condition in terms of a kinematic constraint involving a Dirac delta function.

While details are omitted, the above system of Eqs. (1-3) can be discretized in the following manner to yield a system of linear equations for the velocity, pressure, and boundary forces at the next time $t^{n+1}$ :

$$
\left[\begin{array}{cc}
A & Q \\
Q^{T} & 0
\end{array}\right]\left(\begin{array}{c}
q^{n+1} \\
\lambda
\end{array}\right)=\left(\begin{array}{l}
r_{1} \\
r_{2}
\end{array}\right) .
$$

Here $q^{n+1}$ is the discrete velocity vector and $\lambda=[p, \tilde{f}]^{T}$ is a set of Lagrangian multipliers consisting of pressure and boundary force. The current method highlights the analogous roles of pressure and boundary force in enforcing the kinematic constraints of divergence-free and no-slip on the velocity field. Matrix $A$ is an implicit operator for $q^{n+1}$ and matrix $Q=[G, H]$ is composed of two operators, namely the discrete gradient $(G)$ and regularization $(H)$ operators. The right-hand-side vector, $\left(r_{1}, r_{2}\right)^{T}$, is an explicit term 
based on the old time steps and boundary conditions. The kinematic constraints are represented by the second row of the above equation.

One can then perform an approximate LU decomposition ${ }^{9}$ of Eq. (4) and solve for the flow field in three steps:

$$
\begin{aligned}
A q^{*} & =r_{1}, & & \text { (Solve for intermediate velocity) } \\
Q^{T} B^{N} Q \lambda & =Q^{T} q^{*}-r_{2}, & & \text { (Solve the modified Poisson equation) } \\
q^{n+1} & =q^{*}-B^{N} Q \lambda, & & \text { (Projection step) }
\end{aligned}
$$

where $B^{N}$ is the $N$-th order approximation of $A^{-1}$ in time. The current formulation is similar to the traditional fractional step methods but with two major differences. First, the Poisson equation is modified to simultaneously solve for the pressure and boundary force implicitly. Second, the intermediate velocity vector $q^{*}$ is projected onto the solution space where both divergence-free and no-slip conditions are satisfied. Because the boundary force is determined implicitly without any constitutive relations, the present formulation can use larger CFL numbers compared to some past methods. Symmetry and positive-definiteness of the system are preserved such that the conjugate gradient method can be used to solve for the flow field efficiently. This formulation is found to be second order accurate in time and better than first order accurate in space in the $L_{2}$ sense. $^{8}$

\section{B. Simulation Setup}

Three-dimensional simulations of flow over low-aspect ratio plates are performed in a large rectangular box of size $[-4,6.1] \times[-5,5] \times[-5,5]$ in the streamwise $(x)$, vertical $(y)$, and spanwise $(z)$ directions with a grid size of $125 \times 55 \times 80$. Two-dimensional flow simulations are also considered with a computational domain of $[-15,15] \times[15,15]$ in the streamwise $(x)$ and vertical $(y)$ directions with a grid size of $400 \times 200$. In both cases, the spatial variables are non-dimensionalized by the mean chord $\bar{c}$ of the plate. Grid stretching is applied in all directions with finer resolution near the plate to capture the wake structure as illustrated in Figure 1. To ensure that flow field is well resolved, a finer grid with a size of $150 \times 66 \times 96$ has also been used resulting in less than $5 \%$ difference in the solution for the three dimensional cases. For the two-dimensional cases, similar tests have been performed to verify the grid resolution and domain size.

Boundary conditions along all sides are set to uniform flow $\left(U_{\infty}, 0,0\right)$ except for the outlet boundary where a convective boundary condition $\left(\frac{\partial \mathbf{u}}{\partial t}+U_{\infty} \frac{\partial \mathbf{u}}{\partial x}=0\right)$ is applied. Inside the computational domain, a flat plate is positioned with its center of mass at the origin. This flat plate is instantaneously generated at $t=0$ in an initially uniform flow to model an impulsively started translating plate. Computations are performed to capture the behavior of both the initial transient and the long time flows.

The forces on the flat plate $\left(F_{x}, F_{y}\right)$ are described in terms of the lift and drag coefficients defined by

$$
C_{L}=\frac{F_{y}}{\frac{1}{2} \rho U_{\infty}^{2} A} \quad \text { and } \quad C_{D}=\frac{F_{x}}{\frac{1}{2} \rho U_{\infty}^{2} A},
$$

where $\rho$ is the freestream density of the fluid. We report all spatial and temporal variables in non-dimensional units normalized by the mean chord and the freestream velocity (i.e. $x / \bar{c}$ and $t U_{\infty} / \bar{c}$ ).

Numerical results are compared to experimental measurements for flow over a rectangular flat plate of $R=2$ at $R e=100$. Once the numerical solution is validated, a number of simulations are performed at $R e=300$ for rectangular plates of different aspect ratios for a range of angles of attacks.

Different planform geometries are also considered to investigate the interaction of leading edge and tip vortices. Elliptic, semicircular, and delta-shaped planforms having the same area with the rectangular plate of $R=2$ are selected. 
(a)

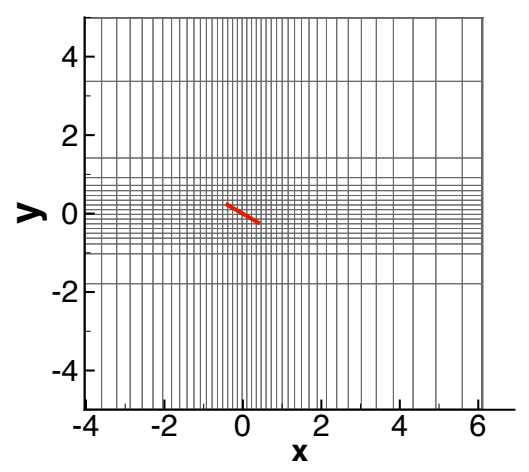

(b)

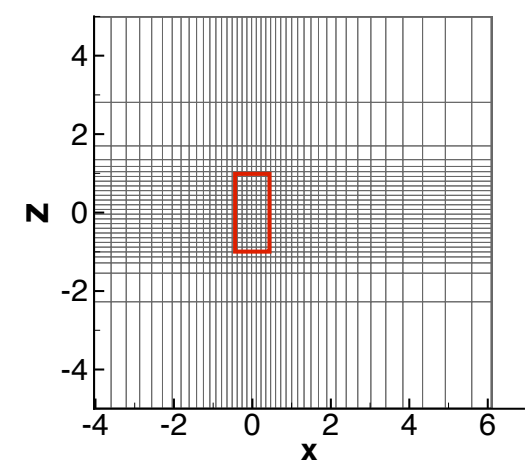

Figure 1. The computational domains with stretched grid used in the three-dimensional simulations shown for the (a) $x y$ - and (b) $x z$-planes (only every third grid is shown). A rectangular plate of $R=2$ at $\alpha=30^{\circ}$ is shown in red for reference.

\section{Experimental Validation}

Companion experiments are performed in a tow-tank $(1 \mathrm{~m} \times 2.4 \mathrm{~m} \times 1.2 \mathrm{~m})$ filled with mineral oil in which a rectangular flat plate is translated. The flat plate is rigidly mounted to a six-axis force sensor at one wing tip to limit lift due to backlash in the gearbox. This setup is attached to a translation sled equipped with a servo motor providing control of the translational velocity. ${ }^{10} \mathrm{~A}$ constant translation velocity is maintained by the plate after an initial swift ramping acceleration from rest.

Stereo digital particle image velocimetry (DPIV) ${ }^{11}$ is used to quantify the flow field around the plate at several spanwise positions with a vector field size of $57 \times 82$. Slices of the flow field are captured from the midspan to $0.22 \bar{c}$ off the wing tip at eleven locations.

To validate the numerical solution, we consider the force on a rectangular flat plate of $R=2$ with $R e=100$ at steady state ${ }^{\mathrm{a}}$ for a range of angles of attack, $\alpha$. Figure 2 compares measured lift and drag coefficients with the simulations. Two sets of numerical results are presented from a case with grid size of $125 \times 55 \times 80$ (fine grid) and that with half the grid resolution (coarse grid). There are some minor differences in the results that are somewhat expected. The experiments are conducted with a finite thickness plate while the numerical simulation is trying to model an infinitely thin plate, and the force on the plate may be influenced by the presence of the sting on one of the wing tips.

Lift coefficients from experiments and simulations are in agreement over a wide range of $\alpha$. The maximum discrepancy of $7.1 \%$ occurs at $\alpha=30^{\circ}$. Drag coefficients show discrepancy between experiment and the coarse simulation that is due to the thickness of the flat plate. In experiments, the plate's thickness is $0.037 \bar{c}$. In the simulations, we attempt to model an infinitely thin plate with regularized body forces. Thus there is an effective thickness that depends on the grid spacing. Simulations with higher resolution (the stars in Figure $2 \mathrm{~b}$ ) provide better agreement for the drag coefficient, with $3.1 \%, 3.9 \%$, and $5.5 \%$ differences at $\alpha=0^{\circ}, 30^{\circ}$, and $90^{\circ}$, respectively ${ }^{\mathrm{b}}$.

Lift coefficients for strictly two-dimensional simulations are also plotted for comparison ${ }^{\mathrm{c}}$. The pronounced decrease in lift due to three-dimensionality (induced drag) is evident. The two-dimensional flow becomes unsteady past $\alpha \approx 25^{\circ}$, resulting in regular vortex shedding. The plus and diamond signs represent the

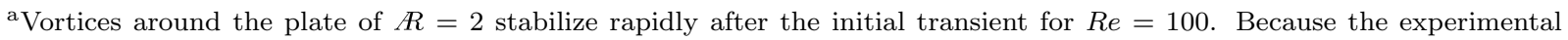
setup limited the maximum travel distance to 13 chord lengths, we consider steady-state only in this subsection to be at $t=13$ for the purpose of validation. Note that the force exerted on the plate decays very slowly past $t=13$ in a minor amount.

${ }^{\mathrm{b}}$ Difference normalized by experimentally measured $C_{D}$ at $\alpha=90^{\circ}$

${ }^{\mathrm{c}}$ We thank Mr. Sunil Ahuja (Princeton University) for providing the two-dimensional data at $R e=100$.
} 
maximum and minimum force exerted on the two-dimensional plate by the periodic vortex shedding. In contrast, the three-dimensional airfoils experience a steady separated flow after an initial transient discussed below.

Next, the vorticity fields are compared with the DPIV data in Figure 3. Comparisons are made at $t=1.5$ and 13 along the mid-span $(z=0)$. Figure 4 illustrates the three-dimensional structure of the spanwise vorticity distribution at $t=1.5$ from both data sets. At this initial stage in time, the LEV is closely attached to the back of the plate providing enhanced lift compared to steady state. The flow fields from the numerical solution and the DPIV data are in good agreement. Based on this validation, we now continue to investigate flows around rectangular flat plates with different aspect ratios and angles of attack.
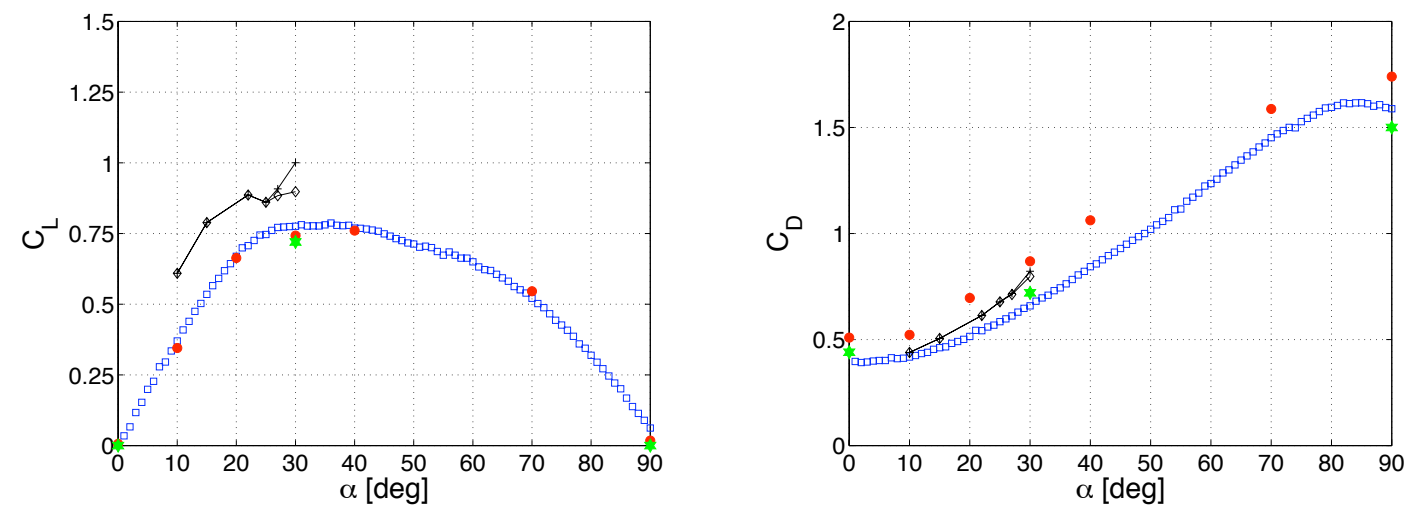

Figure 2. Steady state (left) lift and (right) drag coefficients for rectangular flat plate of $R=2$ for a range of angles of attack at $R e=100$. Results shown from experimental measurements $(\square)$, simulations with coarse grid $(\bullet)$, simulations with fine grid $(\star)$, and two-dimensional simulations (max: + and min: $\diamond)$.

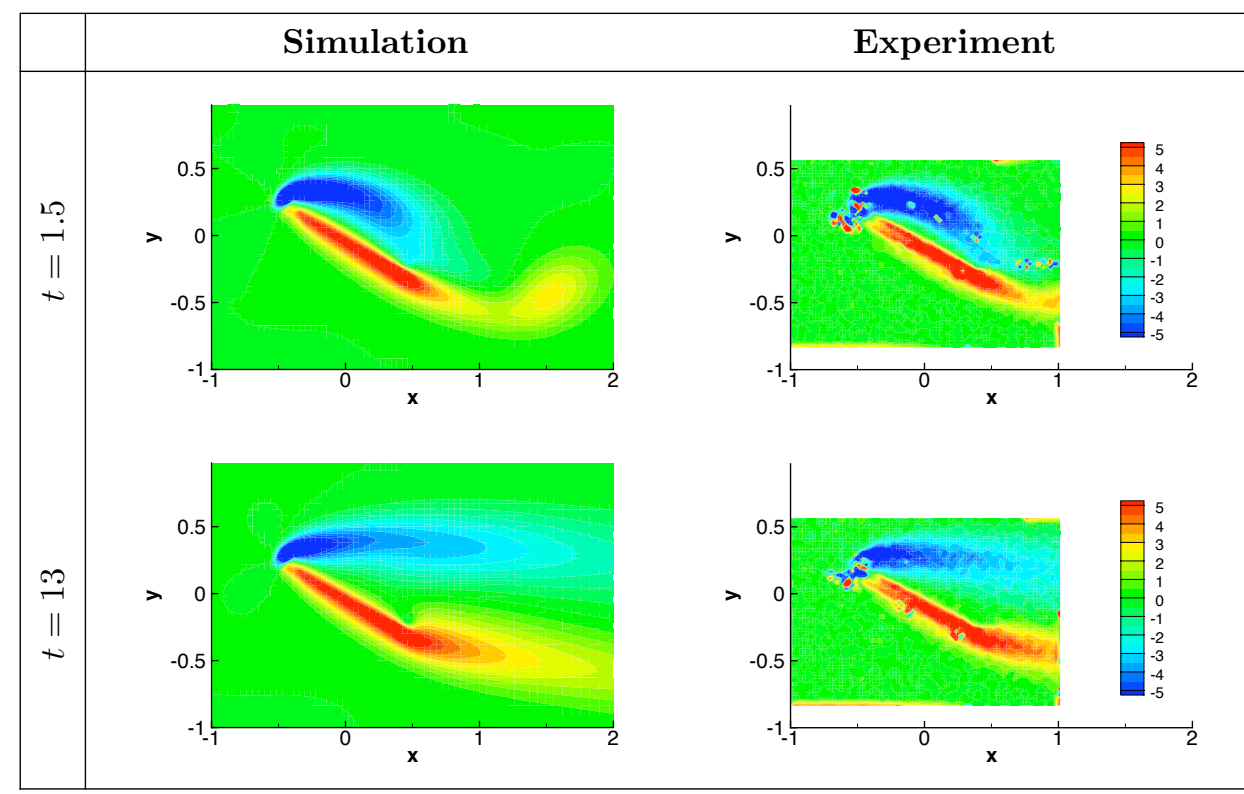

Figure 3. Snapshots of spanwise vorticity $\left(\omega_{z}\right)$ profiles along the midspan $(z=0)$ at $R e=100$ for a rectangular flat plate of $R=2$ based on simulations and the DPIV measurements. 


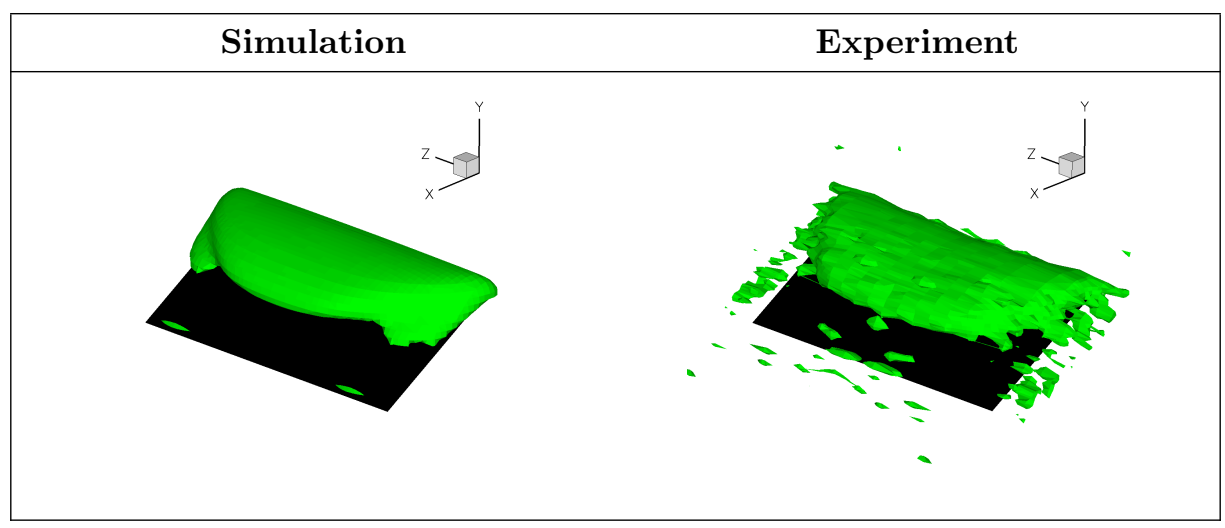

Figure 4. Isosurface for the spanwize vorticity of $\omega_{z}=-4$ at $t=1.5$ illustrating the LEV formation viewing from the rear of the plate $(R=2, R e=100)$.

\section{Results}

\section{A. Flow over Flat Rectangular Plates}

We now investigate the influence of aspect ratio and angle of attack for a slightly higher Reynolds number of $R e=300$. This particular Reynolds number is selected for this study as it is past the critical Reynolds number for flow over a sphere. ${ }^{12}$ First, we consider flow over flat rectangular plates of different aspect ratios $(\boldsymbol{R}=1,1.5,2)$ at an angle of attack of $\alpha=30^{\circ}$ from $t=0$ to 70 . The wake structures behind the plates are captured with several snapshots in time by the vorticity iso-surface as shown in Figure 5. The flow exhibits symmetry about the midspan; attempts to break this symmetry by adding perturbation have not yet been pursued.

Early in time, the distribution of vorticity around the rectangular plate is similar for all three finite aspect ratios. The tip vortices have not yet had much influence on the LEV at this stage. The spanwise vorticity profile presented for $R=2$ at $t=1.5$ in Figure 5 shows the LEV located just above the plate. This profile is observed during the start-up transient for all cases including the two-dimensional wing. The structure is similar to that found in flow over flapping wings. ${ }^{11}$

Lift and drag histories are presented in Figure 6 for angles of attack $0<\alpha<60^{\circ}$. For all three aspect ratios and angles of attack, the lift reaches a maximum around $t=1.5$. The universality of this number is discussed in more detail below. After this time, the LEV begins to lift off the upper surface resulting in a larger, more diffuse separated region (and a significant drop in lift). For the largest aspect ratio $(R=2)$ with $\alpha>15^{\circ}$, the LEV begins to detach around $t=5$, and forms a hairpin vortex that is shed into the wake at later time. These hairpin vortices then repeatedly form, detach, and reform through the simulation. There is no highly pronounced vortex shedding frequency based on a spectral analysis performed at large time up to $t=70$. The angle-of-attack at which this shedding first occurs varies; commencing at $\alpha>15^{\circ}$, $20^{\circ}$, and $30^{\circ}$ for $R=1,1.5$, and 2 , respectively. For $R=2$, the angle at which shedding first occurs approaches that for the two-dimensional $(R=\infty)$ case. Thus the aspect ratio has a significant influence on the wake structure and stability of the LEV.

The behavior of the tip vortices is also different for the different aspect ratios. For $t<8$, their structure is similar for all three finite aspect ratios; they grow by rolling up the vortex sheet generated at the wing tips and trailing edge and develop into long columnar streamwise vortices. However, for the larger aspect ratio of $R=2$ the shedding of hairpin vortices from the leading-edge produces a strong interaction between tip and hairpin vortices that gives rise to the irregular shedding. Conversely, the absence of tip vortices in the two-dimensional cases results in a very regular shedding of the leading-edge and trailing-edge vortices resembling the Karman vortex street behind a circular cylinder. 
Despite their interactions, the leading-edge and tip vortices remain distinct for all three-dimensional cases. The separation of the vortical structures indicates a lack of any convective vorticity flux in the spanwise direction (i.e. from the midspan to the tips). Figure 7 showing the convective flux of spanwise vorticity, $\mathbf{u} \cdot \nabla \omega_{z}$, around the plate clearly indicates that there is effectively no spanwise vorticity convected from the LEV into the tip vortices. Such transport has been shown to give rise to stability of the LEV for flapping wings. ${ }^{11}$ For the translating rectangular wings, there is no mechanism to relieve the vorticity being fed into the $\mathrm{LEV}^{\mathrm{d}}$. Later we consider alternative planform geometries that induce some spanwise flow. Also, in section $\mathrm{C}$, we give some preliminary results on the effect of active control (in the form of a modeled steady mass injection near the leading edge) on the lift generation and LEV stability.

To further contrast the difference between the force exerted by two- and three-dimensional flows, we extract the maximum lift coefficients over time from the initial transients in all cases. The maximum lift for different aspect ratios are plotted as a function of $\alpha$ in Figure 8. Stronger influence of downwash from the tip vortices results in reduced lift for lower aspect ratio plates. For the limiting case of $R=\infty$, the maximum lift is much higher due to the absence of tip effects. However, there is a favorable feature of the tip vortices evident in Figure 8 also. While the two-dimensional curve reaches its maximum around $\alpha=35^{\circ}$, the finite aspect ratio cases achieve their maxima near $\alpha=45^{\circ}$. The offset in the angles of attack is caused by the tip vortices supporting prolonged attachment of the LEV at a higher angle of attack for three-dimensional flows in comparison to the two-dimensional case.

After the initial start-up flow, both lift and drag experienced by the plates are reduced significantly at large time as shown in Figure 9 in terms of the time average quantities. Despite the variation in the maximum lift observed in Figure 8 and the fluctuations seen in Figure 6, the time-averaged forces experienced by the plates are nearly the same. Also the magnitude of these forces are much lower, falling to as much as half the maximum value.

Finally, we call attention to the time at which the maximum lift is achieved. We denote this time by $t^{*}$ and present its value on Figure 10. It is found that over the considered range of finite aspect ratios and angles of attack, $t^{*}$ is fairly constant with a value of $t^{*} \approx 1.5$ because the profiles of the LEV are similar among all cases. As the accumulation of spanwise vorticity generated by the leading edge contributes to the growth of the LEV, there is reminiscence to the formation number used to describe the time at which vortex rings can no longer grow larger in strength. ${ }^{13}$ Since formation number is found to be a universal quantity for a variety of flow, ${ }^{14}$ it is not too surprising that $t^{*}$ is also fairly constant for the number of three-dimensional cases considered here.

In the case of two dimensional flow, we observe a wider range of $t^{*}$ between 1.3 and 2.4 for $\alpha<45^{\circ}$. At higher $\alpha$, a second local maximum startes to emerge for the two-dimensional flow lowering $t^{*}$ significantly.

\section{B. Effect of Planform Geometry}

We now vary the planform geometry at $\alpha=30^{\circ}$ for $R e=300$. Because the rectangular flat plates have straight leading edges inhibiting spanwise transport of vorticity, we consider elliptic, semicircular, and delta planforms to induce spanwise vorticity transport along the leading edge to the tip vortices. The ellipse and delta planforms have $R=2$ and the semicircle has a similar $R=8 / \pi=2.546$. Geometries are chosen such that the area and the mean chord length are identical ${ }^{\mathrm{e}}$ to those of the rectangular plate of $R=2$, which is previously observed to have the most unsteadiness in the wake among the finite aspect ratios considered. We discuss the differences in the wake in contrast to the rectangular flat plate of $R=2$.

Flow behind different planforms is illustrated in Figure 11. Compared to the rectangular profile, the elliptic and semicircular plates generate wake with a less distinct transition between leading edge and tip vortices due to the vorticity transport from the LEV to the tip vortices. Snapshots of an isosurface of $\mathbf{u} \cdot \nabla \omega_{z}$ are presented for the three geometries in Figure 12 at $t=5$. In all three cases, spanwise vorticity is carried away from the leading edge to the tip vortices, which is different from flow around rectangular planforms

\footnotetext{
${ }^{\mathrm{d}}$ Viscous diffusion provides a mechanism to diffuse vorticity out of the LEV, but is apparently too weak at $R e=300$ to stabilize the LEV. In the case of $R e=100$, the LEV is found to be stable at steady state due to viscous diffusion.

${ }^{\text {e}}$ Except for the semicircular wing which only has an identical mean chord length.
} 


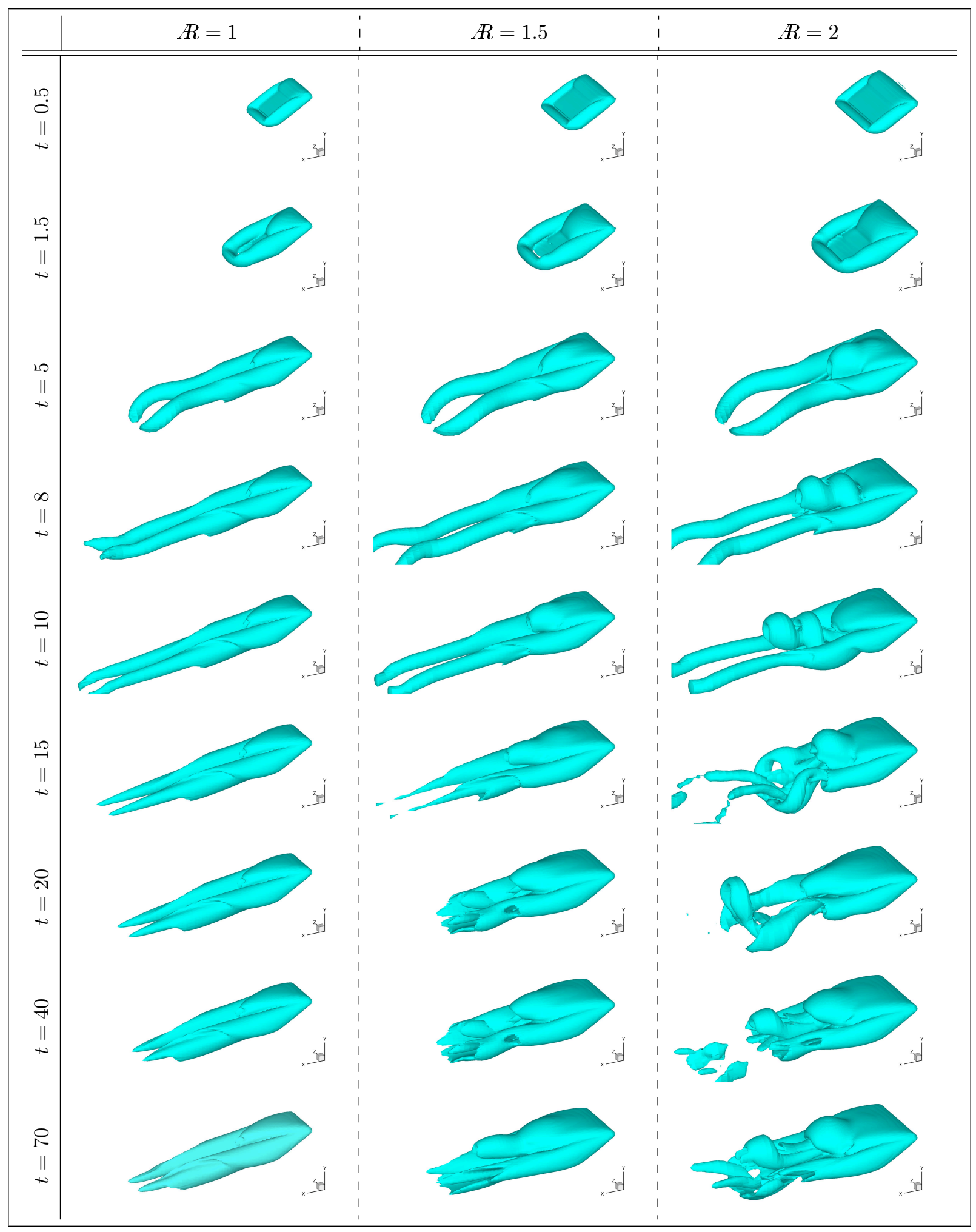

Figure 5. Snapshots of the wake represented by isosurface of $\left\|\omega_{z}\right\|_{2}=2$ behind rectangular flat plates of $R=1$, 1.5 and 2 at $\alpha=30^{\circ}$ and $R e=300$. Viewing downward from the starboard side of the plate.

8 of 16

American Institute of Aeronautics and Astronautics 


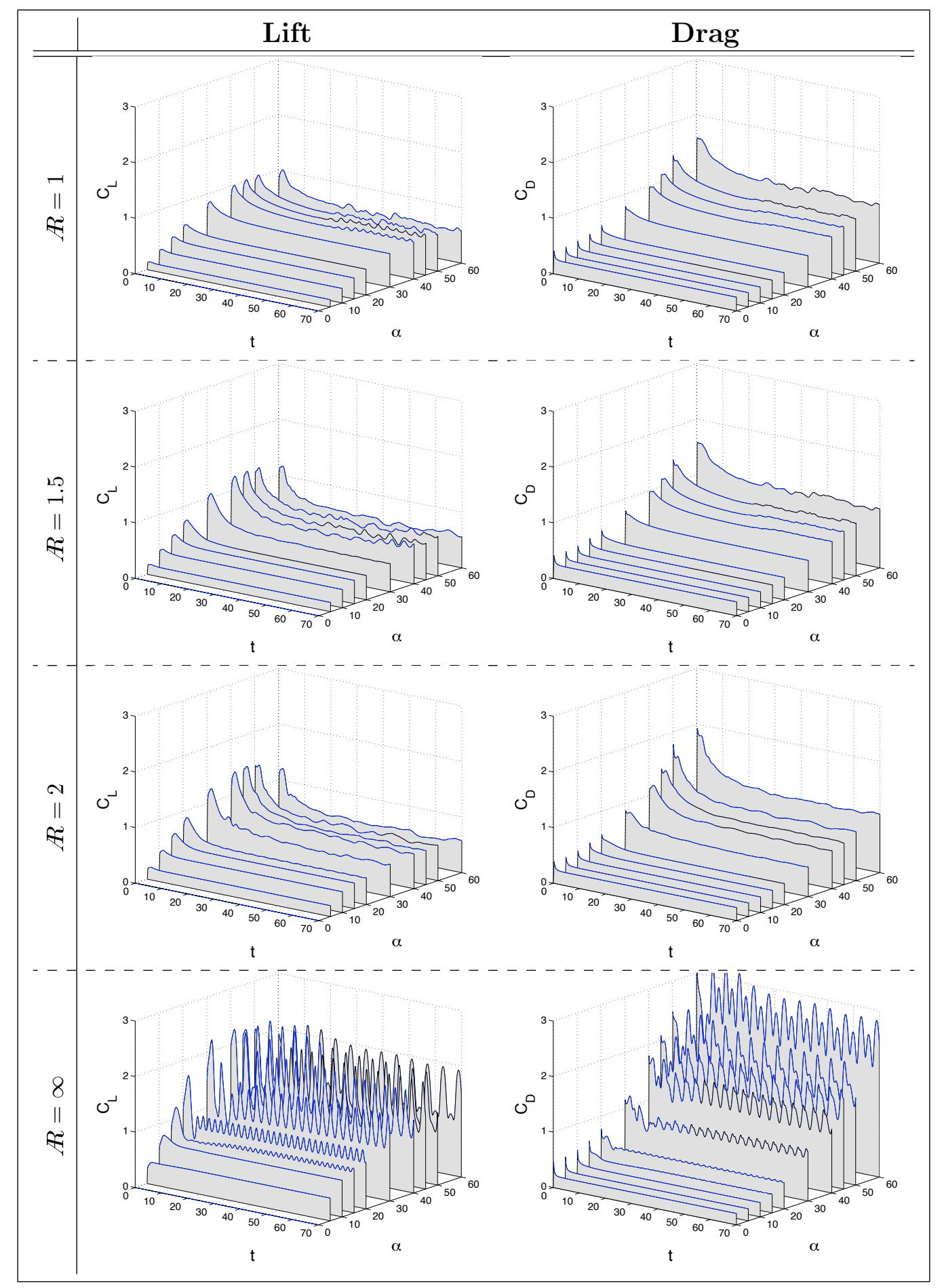

Figure 6. Lift and drag history on rectangular flat plates of different $R$ for a range of angles of attack at $R e=300$ (shown for $\alpha=0,5,10,15,20,30,40,45,50$, and 60 degrees). 


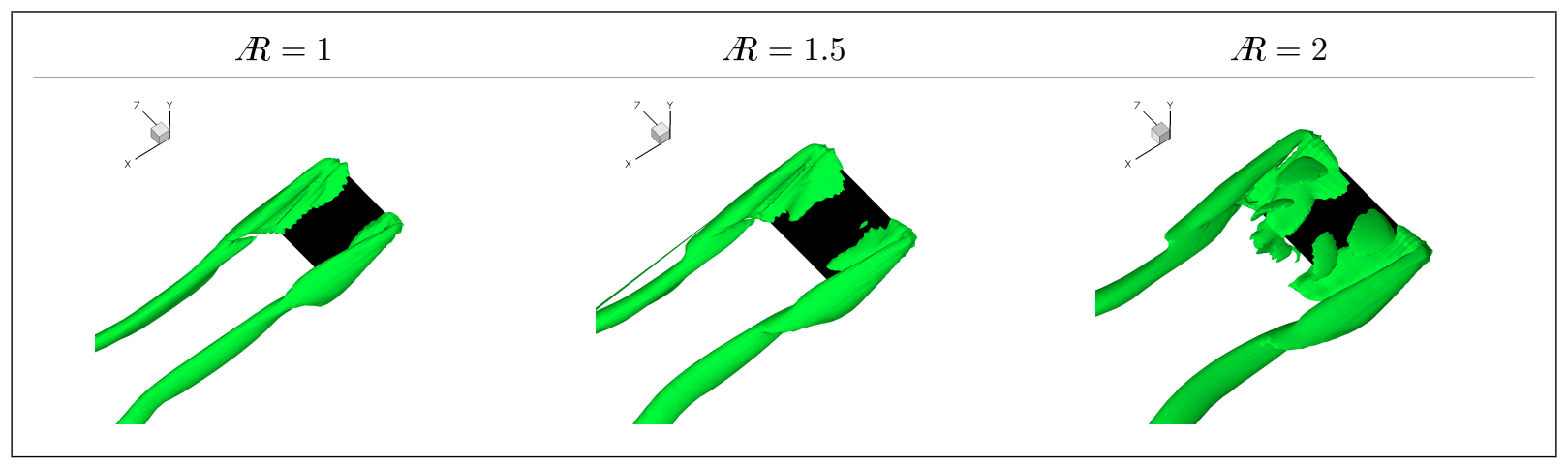

Figure 7. Top view of isosurface shown at $t=5$ for $\mathbf{u} \cdot \nabla \omega_{z}=10$ to illustrate the convection of spanwise vorticity for rectangular planforms with different $R$ s.

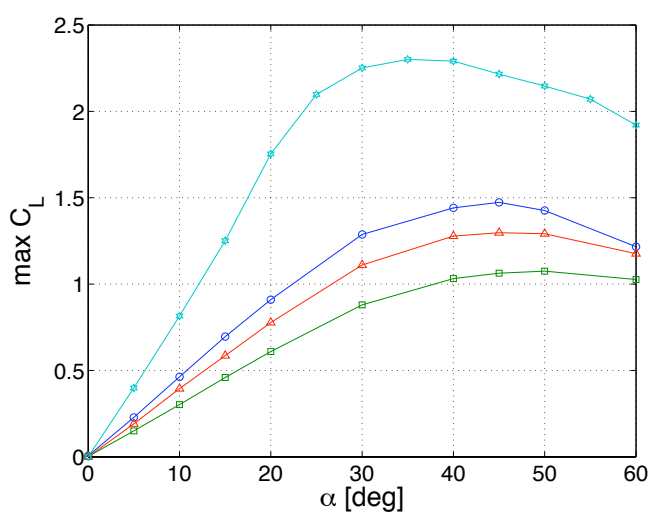

Figure 8. Maximum lift coefficient achieved by rectangular flat plates of $R=1(\square), R=1.5(\triangle), R=2(\bigcirc)$, and $R=\infty(\star)$.
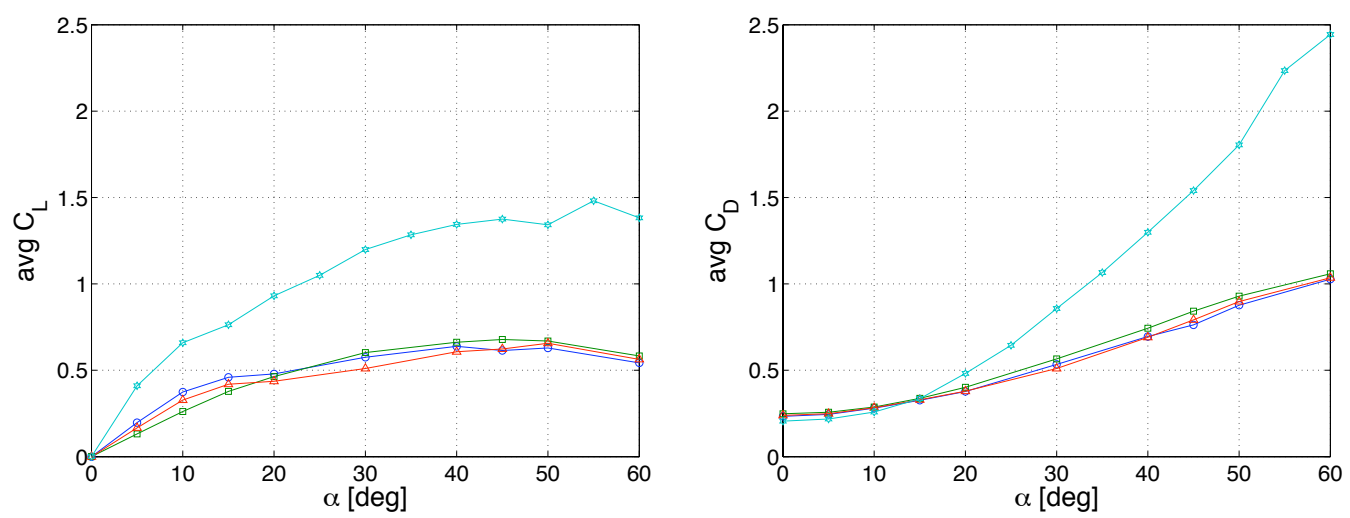

Figure 9. Time average lift and drag coefficients over steady/quasi-steady states for rectangular flat plates of $R=1(\square), R=1.5(\triangle), R=2(\bigcirc)$, and $R=\infty(\star)$.

10 of 16 


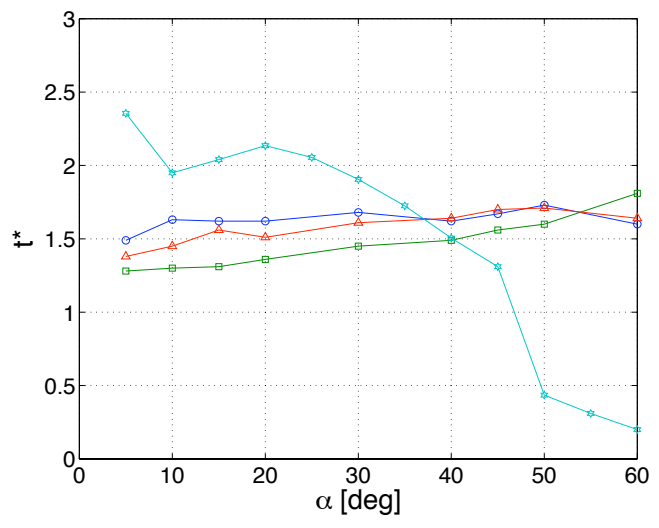

Figure 10. Time at which maximum lift is achieved by rectangular flat plates of $R=1(\square), R=1.5(\triangle), R=2$ $(\bigcirc)$, and $R=\infty(\star)$.

shown in Figure 7. Hence it takes longer time for the LEV to separate from the plate. The leading edge hairpin vortex that is first observed to detach around $t \approx 8$ for the rectangular planform separates at much later time $(t \approx 15)$ for elliptic and semicircular plates (Figure 11). Nonetheless, the overall topologies of the vorticity profile are very similar among the wakes behind the rectangular, elliptic, and semicircular plates.

A notably different distribution of vorticity is obtained for the delta wing with $R=2$ and a sweepback angle of $45^{\circ}$. The absence of wing tips for this planform allows the vortex sheets from the leading edge to roll-up and convect downstream in a very stable manner (see Figures 11 and 12). For this very low Reynolds number of $R e=300$, the LEVs are larger compared to ones from higher Reynolds number flow. ${ }^{15}$ One can observe transient behavior of the wake until $t \approx 15$ in Figure 11. Beyond this point in time, there are some unsteady shedding of small vortical structures behind the rolled up vortices. However, the leading edge vortices do not change its shape much past $t \approx 8$ achieving a near steady state.

The force experienced by different planforms are compared with that exerted on a rectangular plate of $R=2$ over time on Figure 13. The force history is similar amongst the four geometries considered, consistent with the findings of Usherwood and Ellington ${ }^{16}$ for revolving wings at low Reynolds number. Interestingly, the time at which the maximum lift is achieved is again found to be $t \approx 1.5$ for the elliptic and semicircular wings.

The two large fluctuations in the lift coefficient around $t \approx 10$ for the rectangular plate are not present for the other planforms. These two bumps are caused by the two consecutive detachments of leading edge vortices as seen in Figure 5 at $t \approx 8$. The prolonged attachment of the LEV eliminates these features. The vortical structure behind the delta wing does not change much for $t>10$. There is some amount of vorticity shedding into the wake but this occurs much further downstream from the main vortical structure. Most of the vorticity escapes from the wake to the free stream through diffusion in an undisturbing manner. Thus, the force applied on the body does not fluctuate for large time.

\section{Flow over Rectangular Plates with Actuation}

Finally, we consider preliminary results using steady active control to the $R=2$ rectangular plate at an angle of attack of $\alpha=30^{\circ}$. A model actuator represented by a strip of external body force is applied to the flow field expressed by $\mathbf{f} d\left(x-x_{0}\right) d\left(y-y_{0}\right) H(b / 2+z) H(b / 2-z)$, where $d(\cdot)$ is the same regularized delta function used in the immersed boundary method, $H(\cdot)$ is the Heaviside step function, and $b$ is the span of the plate. The direction and the location of the forcing function are defined by the vector $\mathbf{f}$ and $\left(x_{0}, y_{0}, z_{0}\right)$, respectively, where $z_{0} \in[-b / 2, b / 2]$. We set $\mathbf{f}$ to be time-invariant for this study to model steady mass injection near the leading edge.

The magnitude of the forcing function is chosen such that the momentum coefficient of the actuator

11 of 16

American Institute of Aeronautics and Astronautics 


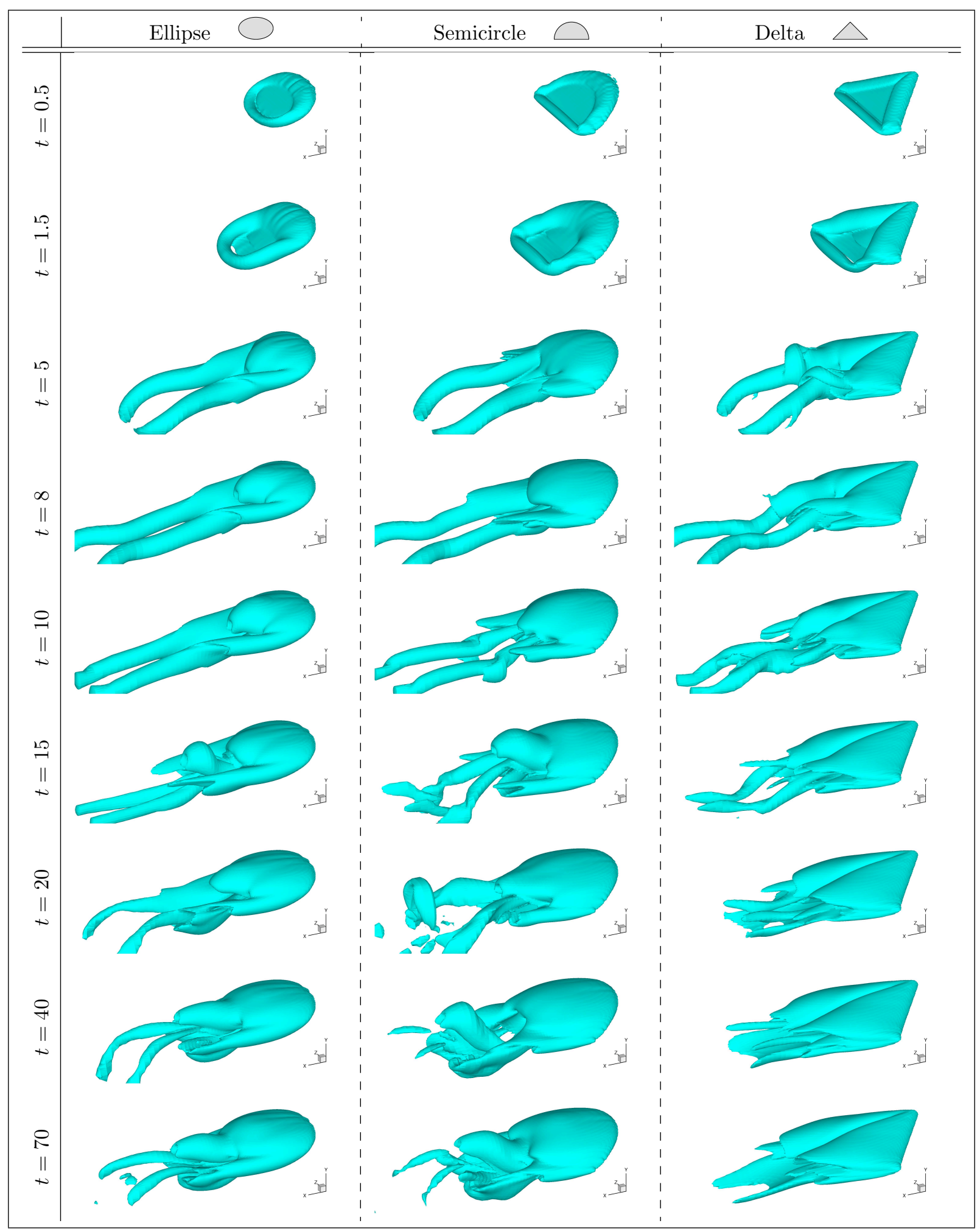

Figure 11. Snapshots of the wake represented by isosurface of $\left\|\omega_{z}\right\|_{2}=2$ behind elliptic, semicircular, and delta-shaped flat plates at $\alpha=30^{\circ}$ and $R e=300$. Viewing downward from the starboard side of the plate.

12 of 16

American Institute of Aeronautics and Astronautics 


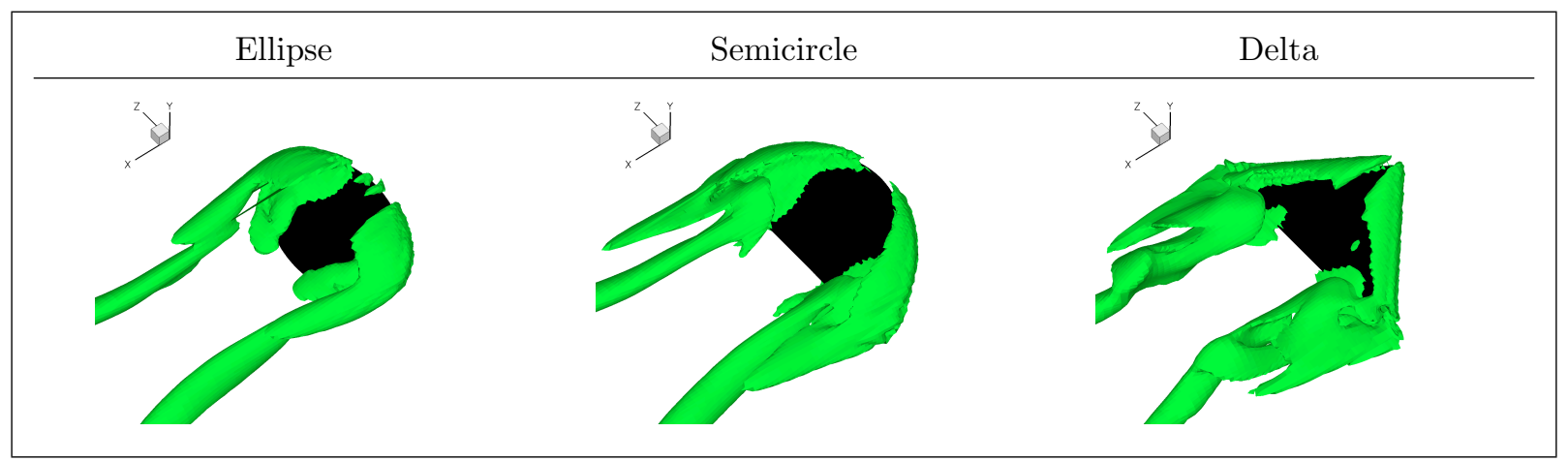

Figure 12. Top view of isosurface shown at $t=5$ for $\mathbf{u} \cdot \nabla \omega_{z}=10$ to illustrate the convection of spanwise vorticity for elliptic, semicircular, and delta planforms.
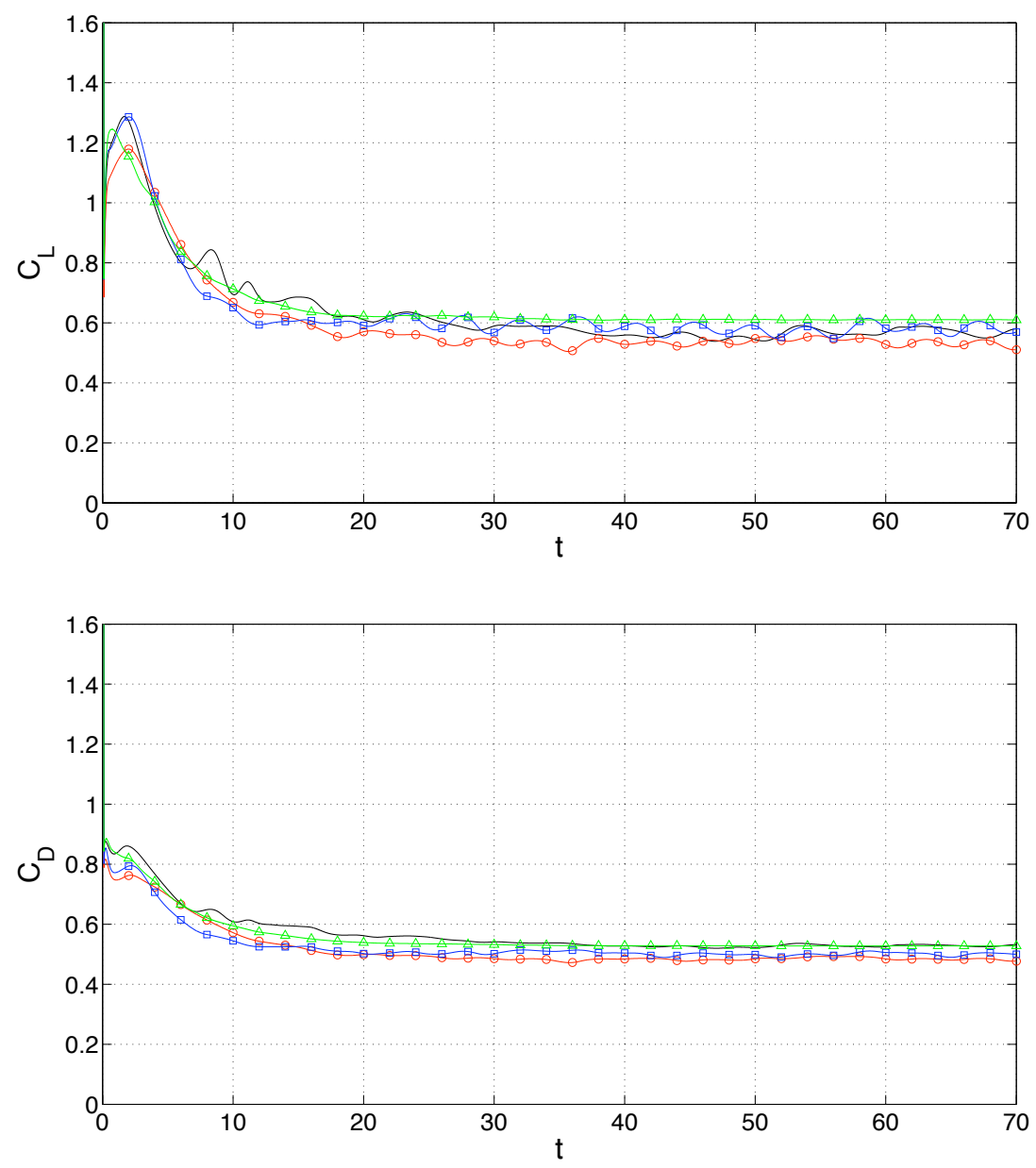

Figure 13. History of lift (top) and drag (bottom) over time with $R e=300$ and $\alpha=30^{\circ}$ for different planform geometries: rectangle of $R=2(\square)$; ellipse ( $\square \bigcirc)$; semicircle $(\square \square)$; and delta $(\square-$ 
model is approximately $c_{\mu} \equiv \rho u_{\text {act }}^{2} A_{\text {act }} /\left(\frac{1}{2} \rho U_{\infty}^{2} A\right) \approx 0.01$ for all cases (here $u_{\text {act }}$ and $A_{\text {act }}$ are the actuator velocity and slot area, respectively). This value is an order of magnitude larger than what is used in real actuators ${ }^{17}$ as this study intends to investigate the fundamental effect of actuation in a preliminary manner. For a more realistic study we would need to use a finer grid to resolve the flow structure in the vicinity of an actuator.

Steady body force is applied $0.1 \mathrm{c}$ above and $0.06 \mathrm{c}$ behind and the leading edge with varied forcing directions. A selection of five forcing angles are made in the streamwise-spanwise $(x-z)$ plane in the directions of (i) downstream, (ii) $45^{\circ}$ angled outward from downstream, (iii) spanwise from the midspan to the tips, (iv) $45^{\circ}$ from upstream, and (v) upstream as illustrated in Figure 14. By choosing such directions, the response of the flow field can be examined for downstream/upstream and spanwise blowing.

(i)

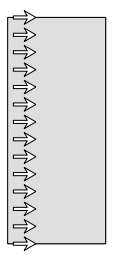

(ii)

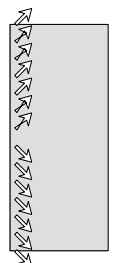

(iii)

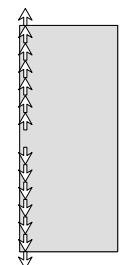

(iv)

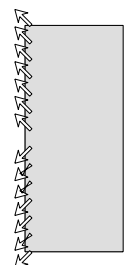

(

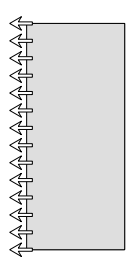

Figure 14. Top view illustration of the actuator directions: (i) downstream; (ii) $45^{\circ}$ from downstream; (iii) spanwise; (iv) $45^{\circ}$ from upstream; and (v) upstream. Free stream is directed from left to right.

Lift and drag history with actuation is presented in Figure 15. Actuation for all five cases does not modify the drag much, but alters the behavior of lift noticeably. Actuation in the streamwise direction enhances the lift by approximately 20 to $30 \%$ compared to the case of no actuation being applied. This is due to the generation of vorticity by the body force that is engulfed into the LEV in addition to what is generated at the leading edge. Blowing upstream provides an opposite effect of reducing the influx of vorticity into the LEV resulting in less lift. The sideway actuation does not change the force on the body in a significant manner.

In future investigation, a better position of the actuator model are to be examined, since it is quite distant from the plate. Feedback control will also be explored. Further simulations with finer resolution are underway to reduce the momentum coefficient and to investigate flow at higher $R e$, where the receptivity of the flow to actuation may be different.

\section{Summary}

We presented results from numerical simulations of flows over low-aspect-ratio flat plates at low Reynolds number using an immersed boundary method. Both the initial transient and long time behavior of the flow was studied by simulating an impulsively started translating plate. Companion experiments were performed in a tow tank to validate the numerical solution with DPIV data and force measurements.

A number of simulations were performed for $R e=300$ with varied aspect ratio, angle of attack, and planform geometry. The aspect ratio was found to have a large influence on the stability of the wake profile and the force experienced by the body. Two-dimensional flows were observed to be vastly different due to the absence of the tip vortices. Behind rectangular plates of $R=1.5$ and 2 , leading-edge vortices were found to form and eventually separate as hairpin vortices. The detached structure would then interact with the tip vortices in a complex manner. Prior to the first separation of the hairpin vortex, the flow profile behind the plates is found to be similar to what has been observed for flapping and revolving wings.

Planform geometries of elliptic, semicircular, and delta were also considered. By providing curvature along the leading edge, convective transport of vorticity from the midspan to the tip somewhat delayed the

14 of 16 

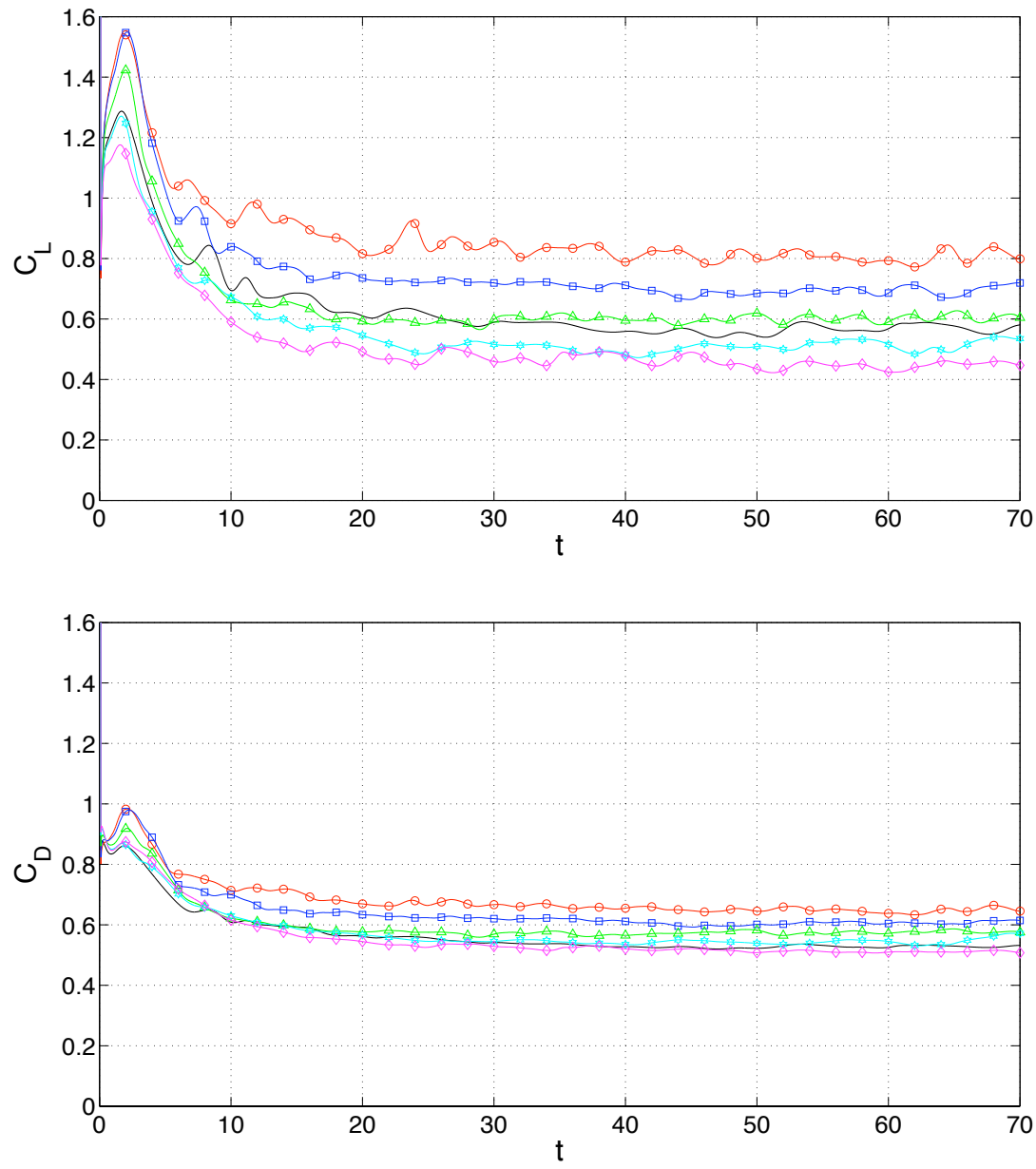

Figure 15. History of lift (top) and drag (bottom) over time for a rectangular plate of $R=2$ at $\alpha=30^{\circ}$ and

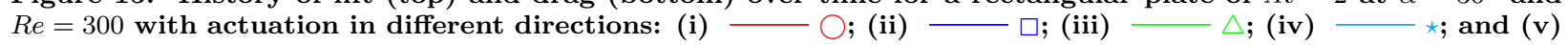
$\diamond$. No actuation shown with 
separation of the LEV. In the case of the delta wing, the leading edge structure was able to remain attached in a very stable manner.

It was observed for most of the cases that maximum lift in time was achieved at a non-dimensional time around $t^{*} \approx 1.5$ regardless of the aspect ratio, angle of attack, and planform geometry. We commented on the reminiscence to the formation number and its possible universality of this non-dimensional time.

Finally, some preliminary results were presented for flow over rectangular plate of $R=2$ with modeled steady mass injection near the leading edge. As an initial study to understand the response of the flow, a rather large momentum coefficient of 0.01 is used. Downstream blowing was found to increase lift but created larger fluctuations over time. Actuation in spanwise and upstream directions were not observed to enhance lift. However, further studies with finer resolution and smaller momentum coefficient for the actuator need to be considered to deduce concrete conclusion.

\section{References}

${ }^{1}$ C. P. Ellington, C. van den Berg, A. P. Willmott, and A. L. R. Thomas, "Leading-edge Vortices in Insect Flight," Nature, Vol. 384, pp. 626-630.

${ }^{2}$ J. M. Birch, W. B. Dickson, and M. H. Dickinson, "Force Production and Flow Structure of the Leading Edge Vortex on Flapping Wings at High and Low Reynolds Numbers," Journal of Experimental Biology, Vol. 207, pp. 1063-1072, 2004.

${ }^{3}$ T. Colonius, C. W. Rowley, G. Tadmor, D. R. Williams, K. Taira, W. B. Dickson, M. Gharib, and M. Dickinson, "ClosedLoop Control of Leading-Edge and Tip Vortices for Small UAV," Conference on Active Flow Control, DFG, Berlin, Sep. 27-29, 2006.

${ }^{4}$ S. Ahuja, C. W. Rowley, I. G. Kevrekidis, T. Colonius, and G. Tadmor, "Low-Dimensional Models for Control of LeadingEdge Vortices: Equilibria and Linearized Models," 45th Aerospace Sciences Meeting and Exhibit, AIAA, (AIAA 2007-709), Reno, NV, Jan. 8-11, 2007.

${ }^{5}$ G. E. Torres and T. J. Mueller, "Low-Aspect-Ratio Wing Aerodynamics at Low Reynolds Numbers," AIAA Journal, Vol. 42, No. 5, pp. 865-873, 2004.

${ }^{6}$ C. S. Peskin, "The Immersed Boundary Method," Acta Numerica, Vol. 11, pp. 479-517, 2002.

${ }^{7}$ R. Mittal and G. Iaccarino, "Immersed Boundary Methods," Annual Review of Fluids Mechanics, Vol. 37, pp. 239-261, 2005.

${ }^{8}$ K. Taira and T. Colonius, "The Immersed Boundary Method: A Projection Approach," Journal of Computational Physics, submitted, 2006.

${ }^{9}$ J. B. Perot, "An Analysis of the Fractional Step Method," Journal of Computational Physics, Vol. 108, pp. 51-58, 1993.

${ }^{10}$ W. B. Dickson and M. H. Dickinson, "The Effect of Advance Ratio on the Aerodynamics of Revolving Wings," Journal of Experimental Biology, Vol. 207, pp. 4269-4281.

${ }^{11}$ C. Poelma, W. B. Dickson, and M. H. Dickinson, "Time-Resolved Reconstruction of the Full Velocity Field Around a Dynamically-Scaled Flapping Wing," Experiments in Fluids, Vol. 41, pp. 213-225, 2006.

${ }^{12}$ T. A. Johnson and V. C. Patel, "Flow past a sphere up to a Reynolds number of 300," Journal of Fluid Mechanics, Vol. 378, pp. 19-70, 1999.

${ }^{13}$ M. Gharib, E. Rambod, and K. Shariff, "A Universal Time Scale for Vortex Ring Formation," Journal of Fluid Mechanics, Vol. 360, pp. 121-140, 1998.

${ }^{14}$ D. Jeon and M. Gharib, "On the Relationship Between the Vortex Formation Process and cylinder Wake Vortex Patterns," Journal of Fluid Mechanics, Vol. 519, pp. 161181, 2004.

${ }^{15}$ I. Gursul, M. R. Allan, and K. J. Badcock, "Opportunities for the Integrated Use of Measurements and Computations for the Understanding of Delta Wing Aerodynamics," Aerospace Science and Technology, Vol. 9, pp. 181-189, 2005.

${ }^{16}$ J. R. Usherwood and C. P. Ellington, "The Aerodynamics of Revolving Wings, I. Model Hawkmoth Wings," Journal of Experimental Biology, Vol. 205, pp. 1547-1564, 2002.

${ }^{17}$ D. Greenblatt and I. J. Wygnanski, "The Control of Flow Separation by Periodic Excitation," Progress in Aerospace Sciences, Vol. 36, pp. 487-545, 2000.

16 of 16 